\title{
Location-Aided Flooding: An Energy-Efficient Data Dissemination Protocol for Wireless Sensor Networks
}

\author{
Harshavardhan Sabbineni, Member, IEEE, and Krishnendu Chakrabarty, Senior Member, IEEE
}

\begin{abstract}
We present a new information dissemination protocol for wireless sensor networks. This protocol uses location information to reduce redundant transmissions, thereby saving energy. The sensor network is divided into virtual grids and each sensor node associates itself with a virtual grid based on its location. Sensor nodes within a virtual grid are classified as either gateway nodes or internal nodes. While gateway nodes are responsible for forwarding the data across virtual grids, internal nodes forward the data within a virtual grid. The proposed approach, termed location-aided flooding (LAF), achieves energy savings by reducing the redundant transmissions of the same packet by a node. We study the performance of LAF for different grid sizes and different node densities and compare it to other well-known methods. We show that LAF can save a significant amount of energy compared to prior methods.
\end{abstract}

Index Terms-Communication protocol, location, energy management, information dissemination, flooding.

\section{INTRODUCTION}

A DVANCES in the miniaturization of microelectronic and mechanical structures (MEMS) have led to batterypowered sensor nodes that have sensing, communication, and processing capabilities [13], [31]. Wireless sensor networks are networks of large numbers of such sensor nodes [10]. These networks are deployed in a target area for collaborative signal processing [33] to facilitate applications such as remote monitoring and surveillance [12], [30], tracking [9], and feature extraction [21]. Other applications of such sensor networks include the monitoring of wild fires, inventory tracking, assembly line monitoring, and networks of bio-sensors for health monitoring.

Sensor nodes are typically characterized by small formfactor, limited battery power, and a small amount of memory. Sensor networks enable distributed collection and processing of sensed data. These networks are usually connected to the outside world with base stations or access points through which a user can retrieve the sensed data for further inference and action. Base stations also send periodic control signals to all the sensor nodes. Such dissemination of information is a challenging problem in sensor networks because of resource constraints. For example, an intruder alert information might need to be forwarded to the entire network. Conventional protocols use classical flooding for disseminating data in a sensor network. Flooding is also used as a preprocessing step in many routing protocols in networks for disseminating route discovery requests [25]. Information dissemination protocols are used in networks for distributing the link state

- The authors are with the Department of Electrical and Computer Engineering, Duke University, Durham, NC 27708.

E-mail: \{hvardhan, krish\}@ee.duke.edu.

Manuscript received 22 Mar. 2004; revised 3 July 2004; accepted 18 Aug. 2004; published online 16 Nov. 2004.

For information on obtaining reprints of this article, please send e-mail to tc@computer.org, and reference IEEECS Log Number TC-0104-0304. information. Routers in the Internet periodically use flooding to update link state information at other nodes [17]. However, flooding suffers from disadvantages such as the broadcast storm problem [6].

In this paper, we present an energy-efficient flooding mechanism, termed location-aided flooding (LAF), for information dissemination in distributed sensor networks. We have designed LAF with the following goals in mind:

- Energy efficiency. Sensor nodes have a very small amount of battery and, hence, any solution must be energy-efficient.

- Self-configuration. Since it is not feasible to have manual intervention for every sensor node, it is preferred that nodes carry out self-configuration.

- Scalable. Sensor networks can typically have hundreds or thousands of nodes, hence any solution for information dissemination should be scalable.

We propose a solution that meets the above design requirements. We present an energy-efficient scheme that uses the concept of virtual grids to partition (self-configure) the set of nodes into groups of gateway nodes and internal nodes. While gateway nodes forward the packets across virtual grids, internal nodes forward the packets within a virtual grid. LAF reduces the number of redundant transmissions by storing a small amount of state information in a packet and inferring the information about nodes that already have the packet from the modified packet header.

Wireless sensor networks are different from ad hoc wireless networks in a number of ways, hence a data dissemination protocol for ad hoc networks does not immediately apply to sensor networks. An excellent survey highlighting the differences between ad hoc networks and sensor networks is presented in [2]. Wireless sensor networks are used for obtaining sensing data from a monitoring area. Sensor nodes send data back to a base station that may be connected to the Internet and where the data 
processing is done. This is typically not the case for wireless ad hoc networks. Ad hoc networks are typically used where there is no fixed infrastructure such as a base station. Ad hoc network routing protocols cannot be directly applied to sensor networks due to lack of scalability and the inability to adapt to a large number of sensor nodes [23]. These factors contribute to a dramatic increase in the control overhead for route discovery, topology updates, and neighbor discovery in ad hoc network routing protocols.

The remainder of the paper is organized as follows: In the next section, we discuss related prior work. In Section 3, we present the details of LAF. The performance evaluation of LAF is presented in Section 4. This section describes the simulation methodology, experimental results, and a comparison with related work. Finally, conclusions and directions for future work are presented in Section 5.

\section{Related Prior Work}

In the classical flooding protocol, the source node starts by sending the packet that needs to be flooded to all of its neighbors [19]. Each recipient node stores a copy of the packet and rebroadcasts the packet exactly once. This process continues until all the nodes that are connected to the source have received the packet. This method of disseminating information is robust to node failures and it delivers the packet to all the nodes in the network provided the network is lossless. Flooding requires that nodes cache the source ID and the sequence number of the packet. This permits the nodes to uniquely identify each packet and prevents the broadcast of the same packet more than once.

A flooding algorithm based on neighborhood knowledge (self-pruning), is presented in [24]. Each node obtains 1-hop neighbor information through periodic Hello packets. Each node includes a list of its one-hop neighbors in the header of each broadcast packet. A node receiving a broadcast packet compares its neighbor list to that of the sender's neighbor list. If the receiving node cannot reach any additional nodes, it does not broadcast the packet. The scalable broadcast algorithm presented in [29] uses 2-hop neighborhood information to limit the number of retransmissions. A node that receives a broadcast packet determines the 1-hop neighbors that need to rebroadcast the packet. A similar approach is taken in the dominant pruning method [40]. This approach uses the header "trail" of the nodes recently visited by the packet to limit the number of broadcasts. It limits the length of the header trail by using a fixed hopcount. In contrast, LAF does not require that each node increase the length of the packet header; only a subset of nodes, referred to as internal nodes and defined in Section 3.5, are required to increase the length of the packet header. Furthermore, LAF uses gateway nodes (also defined in Section 3.5) to limit the header trail rather than using a fixed hop-count.

Information dissemination based on gossiping has been extensively studied in the literature [1], [26], [28]. In [1], gossiping is used to propagate updates among the nodes to maintain database consistency and it is used in [7] to provide reliable multicast. The performance of gossiping for wireless networks is compared with flooding in [15].
SPIN denotes a set of information dissemination protocols for wireless sensor networks [22]. In SPIN, nodes use meta-data to describe the data they possess. Nodes only request the part of data they do not have. Thus, SPIN achieves energy savings by eliminating requests for transmissions of data that nodes already possess. Although SPIN is also a flooding protocol, LAF is different from SPIN in two ways. First, LAF attempts to reduce redundant transmissions by inferring from the packet header about nodes that already have the data, while SPIN uses explicit communication to identify nodes that have the data. Second, while LAF uses location information to assist flooding and reduce energy, SPIN is a generic protocol that does not rely on location information.

Several approaches have been suggested to improve the efficiency of flooding using location information. LAR [37] uses the concept of a request zone to limit the search space for a desired route search. Location information has also been used in one of the five approaches suggested in [6] to contain the broadcast storm problem. A host node suppresses its transmission if the coverage provided by its transmission is less than a certain threshold. This coverage is determined from the locations of other nodes and by calculating the intersecting area of their transmission ranges. Multipoint relaying is proposed to reduce the number of retransmissions due to flooding by choosing a set of relay nodes to broadcast the packets.

The concept of virtual grids in the context of routing is used in GAF [41]. All nodes in a virtual grid are equal from a routing perspective. GAF identifies redundant nodes within each virtual grid and switches off their radios to achieve energy savings. GAF cannot be used for flooding because of the small size of its grids; the density in the network has to be very high for nodes to take advantage of GAF for saving energy. GAF is mainly designed for routing where nodes in a virtual grid maintain the condition that at least one node in the virtual grid is awake. This results in a significant overhead if used for minimizing retransmissions in flooding. However, the goal of LAF is to limit the number of redundant transmissions during data dissemination in the sensor network, hence it differs significantly from GAF.

PAMAS [34] is a multiaccess signaling protocol that conserves battery power by switching off nodes when they are not actively transmitting or receiving packets. It uses a separate signaling channel for transmitting the control messages and for indicating a busy tone when a node is actively transmitting. The power savings are achieved because the signaling channel consumes less power compared to the main radio channel. Since LAF attempts to reduce redundant transmissions, this method can be used in conjunction with PAMAS to achieve higher energy savings.

Several solutions for the broadcast storm problem in flooding [6] have also been proposed. These approaches attempt to reduce the redundant broadcasts by allowing a node to suppress its transmission if some criterion is satisfied after receiving multiple copies of the flooded packet. LAF differs from [6] in a fundamental manner. It uses sender-based control to suppress the redundant transmissions rather than the receiver-based control used in [6] to reduce the redundant transmissions in classical 
flooding. A common feature of all these prior methods is that a node rebroadcasts a packet that all its neighbors have already received.

Finally, an efficient flooding mechanism based on passive clustering for on-demand routing routing protocols is presented in [23]. However, as described in [23], this method also suffers from scalability problems because even simulations are not feasible for networks with over 700 nodes due to excessive control overhead.

\section{LOCATION-AIDED FLOODING}

The proposed protocol, which we describe in this section, uses a variant of classic flooding. We term this variant modified flooding. We describe the basic idea of modified flooding in the next subsection.

\subsection{Modified Flooding}

Modified flooding uses node ids to improve the energy efficiency of information dissemination in wireless sensor networks. Each packet sent using modified flooding includes a special field in the packet header called the Node List. The Node List contains the ids of all the nodes that already have the packet. If we assume the network to be lossless, as is typically done in the related literature, the packet header informs the receiver nodes that all the nodes in the Node List already have the packet, hence forwarding the packet to those nodes is unnecessary.

Modified flooding can be implemented in two ways. One option is to use a unicast scheme in which a sender node sends packets only to intended recipients. This is, however, difficult to implement in wireless networks. A more practical option is to allow all neighbors of the sender node to receive the packet through a broadcast mechanism. A recipient node first checks to see if all its neighbors are already in the Node List. If this test is affirmative, the node does not broadcast the packet. The node also checks to see if its own ID is in the Node List. If this test is affirmative, it does not process the packet and simply drops it. Note that the packet header (described later) contains source ID and sequence number information to facilitate modified flooding. In this work, we assume that the latter approach is used.

As an illustration, we show how the redundant transmissions in flooding can be reduced using modified flooding. When a node $S$ wants to disseminate data to the entire network, it includes the ids of all of its neighbors ${ }^{1}$ in the Node List of the packet header and broadcasts it to all its neighbors. Hereafter, we will refer to the node $S$ as the source of the packet being flooded. A node, say X, after receiving the packet, retrieves the Node List of the packet and compares it with its neighbor list. ${ }^{2}$ If all its neighbors are in the Node List, then $X$ will not broadcast the packet. Thus, redundance transmissions are avoided. Fig. 1 shows the operation of a modified flooding protocol for an example configuration. Node Lists are shown on the communication links. In this figure, node A wishes to flood the network with its sensor data. Therefore, node A broadcasts a packet with its data to all its neighbors.

1. Two nodes are neighbors if they are within the communication range of each other.

2. The neighbor list of a node is the list of ids of all of its neighbors.

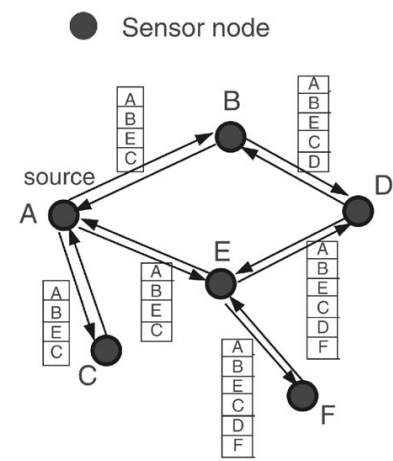

\begin{tabular}{||c|c||c|c||}
\hline \multicolumn{2}{||c|}{ Classical flooding } & \multicolumn{2}{c||}{ Modified flooding } \\
\hline Transmissions & Receptions & Transmissions & Receptions \\
\hline A & B, C, E & A & B, C, E \\
B & A, D & B & A, D \\
C & A & E & A, F, D \\
D & B, E & C & A \\
E & A, D, F & & \\
F & E & & \\
\hline
\end{tabular}

Fig. 1. Example illustrating modified flooding.

Nodes B and E also broadcast the packet, but there are no further broadcasts of this packet.

Although modified flooding results in energy savings by reducing redundant transmissions, the energy savings reduce as the Node List becomes longer; see Fig. 11. In classical flooding, each node broadcasts exactly once and every node receives all the broadcast packets of its neighbors. Hence, this simple network uses six transmissions and 12 receptions to flood the packet. Modified flooding uses four transmissions and nine receptions to flood the same packet. However, if the packet becomes twice as long due to the increase in the length of Node List, this results in an effective number of eight transmissions and 18 receptions. Thus, an increase in the Node List size limits the energy savings and the utility of modified flooding over classical flooding reduces beyond a certain point. To overcome this limitation, we describe our proposed approach, termed location-aided flooding (LAF), in the next section.

\subsection{Location Information}

LAF uses location information to divide the sensor network into virtual grids. This information may be provided by the Global Positioning System (GPS) [16]. In GPS, receivers are used to estimate positions of the nodes in mobile ad hoc networks. However, their high cost and the need for more precise location estimates make them unsuitable for sensor networks. GPS uses atomic clocks for time synchronization. Each GPS satellite transmits signals to sensor nodes on the ground indicating its location and current time. A node estimates the distance to each GPS satellite by estimating the amount of time it takes for the signal to reach the sensor node. Once the distance from four GPS satellites is estimated, a sensor node can calculate its position in three dimensions.

Several other localization schemes are also available in the literature for wireless sensor networks. In [27], a scheme is presented to estimate the relative location of nodes by having 


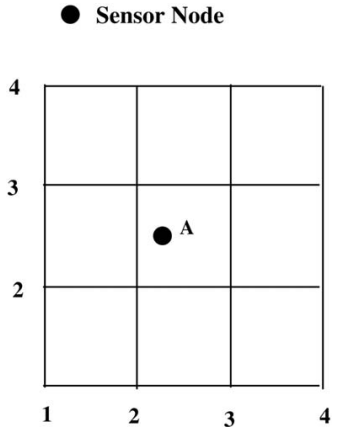

Fig. 2. Example of a virtual grid.

only a few nodes in the sensor network with GPS capability. It uses the received signal strength information (RSSI) as the ranging method to obtain accurate location estimates. Whitehouse and Culler [38] use an ad hoc localization technique called Calamari in combination with a calibration scheme to calculate distance between two nodes using a fusion of RFbased RSSI and acoustic time of flight (TOF). Acoustic ranging [14] can be used to used to get fine-grained position estimates of nodes. Clouqueur et al. [12] propose a low-cost localization technique that uses time-of-arrival ranging. Recursive schemes such as [3] can also be used to get finegrained position estimates of sensor nodes with error within $0.28 \mathrm{~m}$ for nodes of $40 \mathrm{~m}$ radio range.

For our initial discussion, we assume that each node knows its physical location accurately. However, we later show in Section 3 and Section 4 that LAF can tolerate moderate errors in location estimation as well as correlated large errors.

\subsection{Virtual Grids}

LAF divides the monitored area (sensor field) into "virtual grids." Each node associates itself with a virtual grid depending on its physical location. This is illustrated in Fig. 2, where the monitored area is divided into nine virtual grids. Node A belongs to the virtual grid with the bottomleft-corner coordinates $(2,2)$.

\subsection{Packet Header Format}

The header format of the packets used in LAF is shown in Fig. 3. It consists of the sourceID as well as the SeqNumber of the packet. The recoNodeList field is of variable length and contains the list of the nodes that have already received the packet. gridID, the ID of the grid in which the sender of the packet is currently in, and nodeType, whether the node is a gateway node or an internal node, are the other fields. The field gridID is used only by the gateway nodes and is used for preventing the retransmission of packets the grid has already seen. The number of bytes for each field is best determined by the application designer. For example, the typical number of nodes in a sensor network application determines the number of bytes that needs to be reserved for the gridID field.

The size of a virtual grid and the appropriate number of virtual grids depend on the specific application requirements and also on the packet size. If the packet size is small, the overhead of the control data becomes significant after a few rounds of modified flooding. In this paper, we assume that the number of virtual grids is determined a priori.

\section{SourceID SeqNumber recvNodeList GridID nodeType}

Fig. 3. Packet header format in LAF.

\subsection{LAF Node Types}

LAF classifies each sensor node into one of the two types.

- Gateway Nodes. If any of the neighbors of a node A belong to a different virtual grid than that of $\mathrm{A}$, then A becomes a gateway node.

- Internal Nodes. If all the neighbors of a node A belong to the same virtual grid as that of A, then A becomes an internal node.

Nodes determine their virtual grid and status (gateway node or internal node) autonomously using the knowledge of their location information after deployment. This is the case for the example virtual grid shown in Fig. 4. Nodes A, G, F, I, D, and H are gateway nodes while B, C, and E are the internal nodes. Gateway nodes forward the data across virtual grids and internal nodes forward the data within a virtual grid.

\subsection{Information Dissemination Using LAF}

Data forwarding by gateway nodes. When a gateway node receives a packet from within its virtual grid, it checks to see if any of its neighbors within the same virtual grid have not yet received the packet. This is done by comparing the Node List of the packet with the neighbor list of the node. If such nodes exist, then the gateway node appends the ids of those nodes to the Node List of the packet and forwards it to the neighbor nodes that still have not received the message. When a gateway node receives a packet from another gateway node, it strips the packet of its Node List and adds its own id and all its neighbors' ids and forwards the packet to all its neighbors. Thus, the packet becomes shorter as it moves across the virtual grids and increases in size as it moves within a virtual grid. The basic idea behind LAF is to reduce the redundant transmissions by including the node ids in the packet. Virtual grids are used to limit the packet size. Gateway nodes in LAF

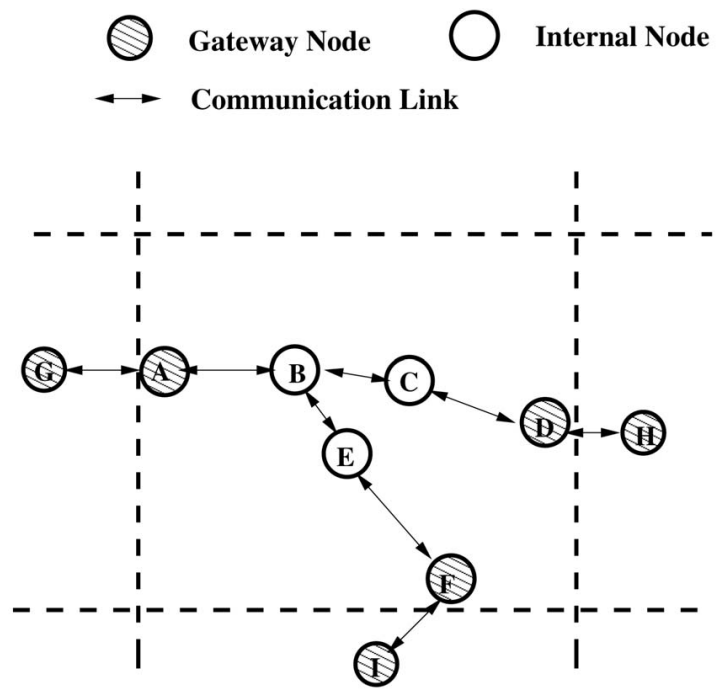

Fig. 4. Illustration of gateway nodes and internal nodes in a virtual grid. 
cache the sourceID and SeqNumber fields of the recently seen packets. This allows the gateway nodes to prevent looping of packets in the network.

Data forwarding by internal nodes. When an internal node receives a packet, it modifies the Node List of the packet. It includes the ids of all its neighbors in the Node List and forwards it to its neighbors if they have not already received a message.

LAF is a simple protocol designed for lossless networks. However, it can be easily adapted for networks with errorprone communication links. Nodes can retransmit a packet multiple times to compensate for the lossy communication links. Note also that LAF does not rely on a uniform placement of nodes. It can be easily used for a sensor network with "holes" because a regular virtual grid can be easily generated in a distributed manner for such a deployment.

\subsection{Resource Management in LAF}

LAF can be made resource-adaptive. When the remaining energy on the various nodes is different, nodes with less available energy can choose to wait for a time-out period before retransmitting the packet that needs to be flooded. This time-out can be preset depending on the application requirements. The key idea behind this is that nodes with less remaining energy should participate only in the high priority tasks of the application, leaving the low-priority tasks to the nodes with high remaining energy. (The alternative is to let all nodes participate to the same extent for all the tasks; however, this causes nodes with less remaining energy to die sooner.) This leads to a better utilization of the network for a longer period.

LAF does not specify a resource management policy and leaves it to the application to choose an appropriate policy depending on its latency and network lifetime and other application-oriented requirements.

Grid Maintenance Costs. Sensor nodes can use any unique attribute of their virtual grid as gridID. In our simulator, nodes use $(x, y)$ as a gridID, where $x$ is the $\mathrm{x}$-coordinate of the top left corner of the virtual grid and $\mathrm{y}$ is the y-coordinate. We estimate the cost of maintaining a grid as follows: Suppose the packet header size is $h$ bits and the packet size is $S$ bits. Also, suppose that the total number of $n$ nodes are present in the monitored region divided into $N$ grids. Suppose the number of beacon messages needed to know the position of a sensor node is $n_{B}$ and the energy needed to receive each beacon message is $E_{B}$. If each node needs $n_{p}$ processing cycles to calculate grid association, then the total energy required to calculate the grid association is $n_{p} \times E_{p}$, where $E_{p}$ denotes the amount of energy needed for a single processing cycle. Hence, the total amount of energy needed for maintaining the grid is $n \times\left[n_{B} E_{B}+n_{p} E_{p}\right]$. Thus, the grid maintenance cost grows only linearly with the size of the network.

\subsection{Completeness of the Data Dissemination Procedure}

In this section, we prove the completeness of LAF as a flooding mechanism. In other words, we show that data flooding can always be accomplished using LAF. A node that wants to flood the network with a data packet becomes the source for that data packet. We prove that if a node receives the data packet from the source through classical flooding, it will also receive the data packet through LAF.

Lemma 1. If a gateway node in a virtual grid receives the packet, then all the nodes in the virtual grid will ultimately receive the packet provided that they received the message using the classical flooding protocol.

Proof. Each node in a virtual grid can be either a gateway node or an internal node. Now, consider a node $\mathrm{A}$ in the virtual grid. Let us denote the neighborhood of $\mathrm{A}$ as the set $N_{A}$ that consists of all the neighbors of A. Consider a node $\mathrm{B}$ in $N_{A}$ that has the message. If the Node List received by $B$ does not contain $A$, then $B$ will forward the message to A. However, the Node List of the packet will contain the id of node A only if node A has the packet according to the LAF protocol. Thus, node A either has the message or it will receive the packet from node $B$. Once node A receives the packet, it will forward it to all its neighbors that still have not received it. Thus, eventually, all the nodes in the grid will receive the packet.

Theorem 1. If a source node floods the network with a message and if LAF is used by every node that forwards the message, then the message reaches every node in the network provided that the message reaches every node in classical flooding.

Proof. We prove the theorem by contradiction. Consider a node in the random network that receives the message using flooding protocol but not using LAF. We call the node that is the originator of the message the source node and the node under consideration the destination node. Also, we refer to the virtual grid in which the source node resides as the source virtual grid and the virtual grid in which the destination node resides as the destination virtual grid. Since the destination node has received the packet in flooding, there exists a path from the source to the destination. That the destination node has not received the packet under LAF implies that none of the gateway nodes in the virtual grid of the destination have received the message (Lemma 1). If any of the gateway nodes received the message, they would have forwarded it to the destination node. This means that none of the neighboring virtual grids of the destination virtual grid received the message. If any of the neighboring grids received the message, they would have forwarded it to the gateway nodes of the destination virtual grid. By continuing in a similar fashion, we can show that the gateway nodes of the source virtual grid also did not receive the message. This implies that no message has been flooded in the source virtual grid, which is a contradiction. Hence, if each node in the network executes the LAF protocol, every node eventually will receive the flooded packet.

Corollary 1. If node failures can only occur before flooding begins, the degree of fault tolerance of the network for LAF is the same as that for classical flooding.

Suppose that some nodes in the network have failed prior to flooding. From Theorem 1, we know that if a message reaches a destination by classical flooding in the network 
with failed nodes, it will also reach the destination node by LAF. Thus, the fault tolerance of LAF can be trivially shown to be equal to that of classical flooding.

An interesting open problem is to compare the fault tolerance of LAF with that of classical flooding if nodes fail during flooding. This problem needs to be investigated in more detail and it is left for future work.

\subsection{Analysis}

In this section, we first study two simple topologies and analyze the energy savings achieved by LAF compared to classical flooding. Then, we derive equations for obtaining the energy savings due to LAF in random networks. Suppose the average size of a data message is $S$ bits and the diameter of the network is $D$. (The diameter of a graph is the longest of the shortest paths between any two nodes.) If $E_{T}$ is the amount of energy needed to transmit one bit of data and $E_{R}$ is the amount of energy needed to receive one bit of data, the amount of energy consumed by a node sending the data message with $k$ node ids and one of its neighbors receiving the message is $(S+k i) E_{T}+(S+k i) E_{R}$, where $i$ denotes size of the node id in bits. For a network of $N$ nodes with a fully connected topology, for each packet that needs to be flooded, there are $N$ transmissions and $N(N-1)$ receptions. Therefore, the energy $E C_{C F}$ consumed in the network is

$$
E C_{C F}=S \times N \times E_{T}+S \times N(N-1) \times E_{R} .
$$

In $\mathrm{LAF}$, since the message is transmitted with the node ids of all the nodes in the network, there will be one transmission and $(N-1)$ receptions. If we ignore the small increase in packet length in LAF, the total energy $E C_{L A F}$ consumed in flooding the packet is

$$
E C_{L A F}=S \times E_{T}+S \times(N-1) \times E_{R} .
$$

For values of $N=30, S=64$ bytes, $E_{T}=0.8 \mu \mathrm{J} / \mathrm{bit}$ and $E_{R}=0.6 \mu \mathrm{J} / \mathrm{bit}$, classical flooding consumes $280 \mathrm{~mJ}$ while LAF consumes approximately $9 \mathrm{~mJ}$ of energy.

As a second example, consider a line topology with $N$ nodes. Each node has at most two neighbors. In this topology, for a message to be flooded, $N$ transmissions and $2 N-2$ receptions are needed. This is due to the fact that, in flooding, each node has to broadcast the packet exactly once and this results in $N$ transmissions. As each node has to listen to all the transmissions of its neighbors, there are a total of $2 N-2$ receptions. Therefore. the energy $E C_{C F}$ consumed in flooding the message is

$$
E C_{C F}=N \times S \times E_{T}+(2 N-2) \times S \times E_{R} .
$$

In LAF, the message length is increased by one each time a node forwards the message in the line topology. A node will not process the message if it is included in the Node ID list. Hence, there are only $N-1$ transmissions and $N$ receptions, the additional reception due to the $N$ th node, that receives the packet but need not transmit it as there are no neighboring nodes that have not yet received the packet. The total energy $E C_{L A F}$ consumed in this case is

$$
\begin{aligned}
E C_{L A F}= & ((1+2+\ldots+N-1) \times i+(N-1) \times S) \\
& \times\left(E_{T}+E_{R}\right) \\
= & \left(N \times \frac{(N-1)}{2} \times i+(N-1) \times S\right) \\
& \times\left(E_{T}+E_{R}\right)+((N-1) \times i+S) \times E_{R} .
\end{aligned}
$$

For values of $N=30, S=64$ bytes, $E_{T}=0.8 \mu \mathrm{J} / \mathrm{bit}, E_{R}=$ $0.6 \mu \mathrm{J} / \mathrm{bit}$, and $i=1$ byte, classical flooding consumes $12 \mathrm{~mJ}$ while LAF consumes approximately $6 \mathrm{~mJ}$ of energy.

Next, we analyze the energy savings in the case of a random network constructed as follows: Nodes are placed at random in a rectangular area. Nodes are battery-powered and have only a limited range for transmission. Two nodes are neighbors if they are within the transmission range $r$ of each other. This type of random network is useful for modeling a large number of practical situations involving ad hoc and sensor networks. Now, we derive equations that predict the energy savings for the LAF scheme. Consider a random network with a total number of $N$ nodes and with $n$ nodes in each virtual grid. Suppose each node has $\Delta$ neighbors on average and $M$ neighbors on average already have the packet. The increase in packet length due to the addition of node ids is considered negligible in comparison with the total packet length. In LAF, a node does not process a packet if it is included in the Node ID list. The amount of energy consumed in flooding the virtual grid $E_{V}$ using modified flooding is then given by

$$
E_{V}=\left[E_{T}+E_{R} \times M\right](n) \times S .
$$

Hence, the total energy $E C_{L A F}$ consumed in flooding the packet throughout the network is

$$
E C_{L A F}=\frac{N}{n} \times E_{V}
$$

In the case of classical flooding, the total energy $E C_{C F}$ consumed is given by

$$
E C_{C F}=\left(E_{T}+E_{R} \times \Delta\right) \times N \times S .
$$

Fig. 5 compares the energy consumed by classical flooding with LAF with varying $\Delta$ and for different values of $\mathrm{M}$, for $N=100, n=10, E_{T}=0.8 \mu \mathrm{J} / \mathrm{bit}, E_{R}=0.6 \mu \mathrm{J} / \mathrm{bit}$, and $S=$ 64 bytes.

\subsection{Errors in Location Estimates}

In the above discussion, we assumed that each node knows its geographical location precisely. However, there might be errors in the location estimate provided to the nodes by GPS [16] or other localization systems [3], [14], [27], [38]. Nevertheless, we do not expect the inaccuracies in position estimation to affect the performance of LAF. This is due to several reasons. First, LAF uses location information to associate a node with a specific grid. If the error in location estimate causes the node to assume a different location in the same grid, it will not affect the functioning of the node from a data dissemination viewpoint. Second, if the error in location estimate causes the node to assume a different virtual grid than the virtual grid it really belongs to, then the node becomes a gateway node in the assumed virtual grid and this also does not affect the performance of LAF significantly. Similarly, if a large correlated error causes a 


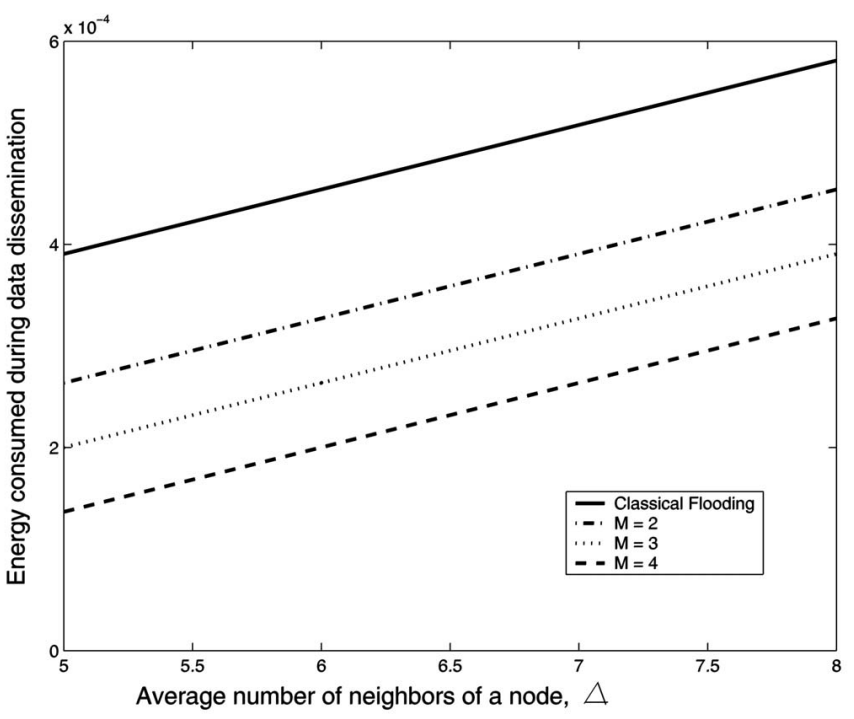

Fig. 5. Energy consumption for LAF (analytical result).

group of nodes belonging to a single virtual grid to be shifted to a different physical location, then the performance of LAF remains unaffected as all the nodes still belong to the same virtual grid.

\section{Performance Evaluation}

We have developed a simulator in $\mathrm{C}++$ to evaluate the performance of LAF and compare it with alternative data dissemination algorithms. We found that LAF protocol achieves higher energy savings compared to both classical flooding and pruning-based methods while disseminating the data with comparable delay. We also found that the nodes with a higher degree (i.e., nodes with more one-hop neighbors) disseminate more data per unit energy in both LAF and modified flooding compared to classical flooding. Thus, dense sensor networks are likely to benefit more from using the LAF protocol for data dissemination in terms of energy savings.

\subsection{Energy Model}

Each sensor node is assumed to have a radio range of $20 \mathrm{~m}$. The bandwidth of the radio is assumed to be $20 \mathrm{Kbps}$. The sensor characteristics are given in Table 1 . These values are taken from the specifications for the TR1000 radio from RF Monolithics [4].

TABLE 1

Radio Characteristics [4]

\begin{tabular}{|l|c|}
\hline Radio Mode & Power Consumption $(\mathbf{m W})$ \\
\hline Transmit $\left(T_{x}\right)$ & 14.88 \\
\hline Receive $\left(R_{x}\right)$ & 12.50 \\
\hline Idle & 12.36 \\
\hline Sleep & 0.016 \\
\hline
\end{tabular}

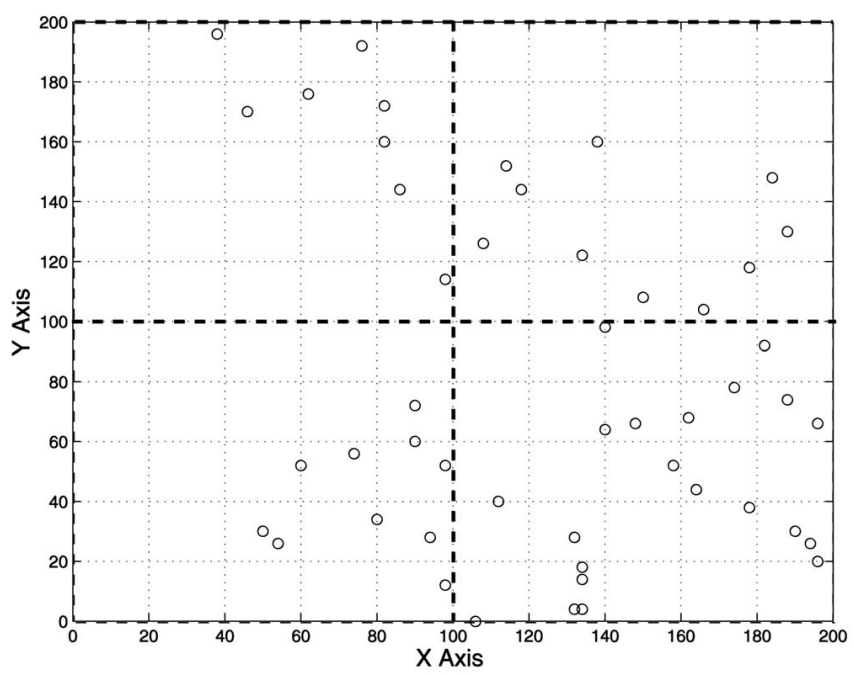

Fig. 6. Test network used in the simulations.

\subsection{Simulation Model}

We initially used a 50-node network in a $200 \times 200 \mathrm{~m}$ monitoring area, as shown in Fig. 6. The monitored area is divided into four virtual grids and has an average of nine gateway nodes. This network is randomly generated with the precondition that the graph be completely connected. The processing delay for transmitting a packet is chosen randomly between $0 \mathrm{~ms}$ and $5 \mathrm{~ms}$. This does not consider the queuing delays and other data processing delays that are incurred. We ran the data dissemination protocols 200 times and averaged the results. In each run, a randomly selected node floods the network with a 64-byte packet. Each node broadcasts a 5-byte HELLO packet every 2s. We have not implemented a localization system in our simulator. Instead, to compare energy consumption by LAF more accurately, we simulate the reception of three 10-byte beacon messages by the nodes every 2 s. Finally, we assume that the network is lossless.

Although LAF relies on a localization scheme, we do not consider it in our simulator for simplicity. Instead, we make use of the geographic locations of sensor nodes provided by our simulator to determine the type of each sensor node (in practice, nodes determine their states autonomously). However, since the message overhead due to LAF is negligible, we believe that this does not affect the results significantly.

\subsubsection{Data Acquired in the System with Time}

Fig. 7 shows the percentage of data disseminated in the system with the passage of time for classical flooding, modified flooding, LAF, self-pruning [24], and dominant pruning [40]. As shown in the figure, the difference in message delay between these protocols is negligible. Fig. 8 shows a zoom-in view of Fig. 7. The difference in delay in these two protocols is visible in Fig. 7. This delay difference can be considered negligible for all practical purposes. The small difference in time delay arises due to an increase in message length in LAF and pruning-based methods and the corresponding increase in propagation time. 


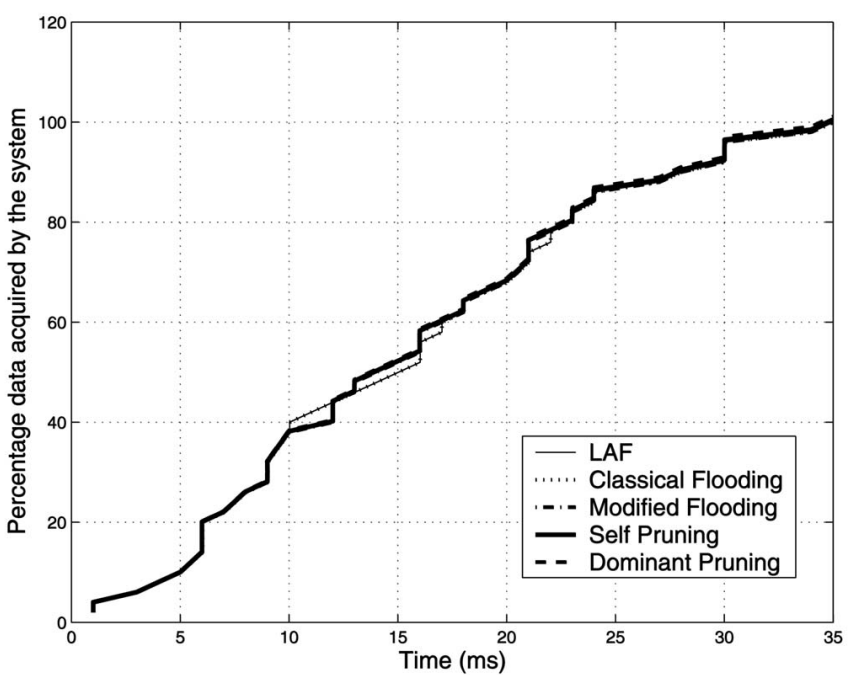

Fig. 7. Data disseminated in the system with time.

\subsubsection{Energy Dissipated in the System with Time}

Next, we measured the energy consumed in the system when these protocols are used for data dissemination purposes. Fig. 9 shows the total energy consumed in the system with time as data gets disseminated in the system. As shown, LAF achieves significant energy savings compared to the flooding protocol. (The energy consumption for LAF is less than $20 \mathrm{~mJ}$ even after $35 \mathrm{~ms}$.) By using a small amount of state information, LAF reduces the number of redundant transmissions significantly.

\subsubsection{Impact of Number of Grids}

We have varied the number of virtual grids for the test network shown in Fig. 6 and evaluated the performance of LAF using our simulator. Fig. 10 shows the energy consumption for the system when the monitored area is divided into 1, 4, 8, and 50 virtual grids, respectively. The energy dissipated in the system decreases with an increase in the number of virtual grids up to a certain point, after which it decreases. This can be explained intuitively as

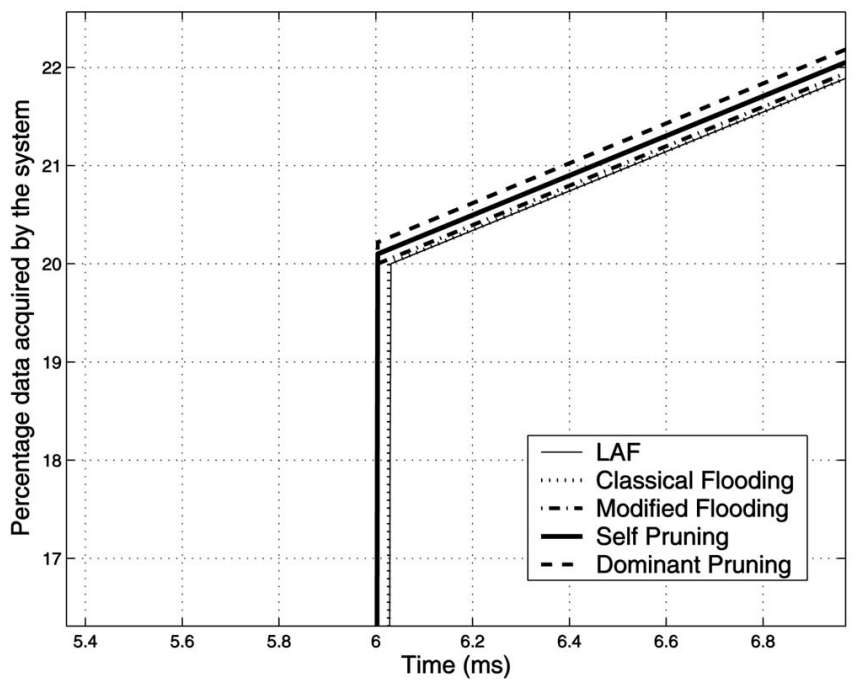

Fig. 8. A zoom-in view of Fig. 7.

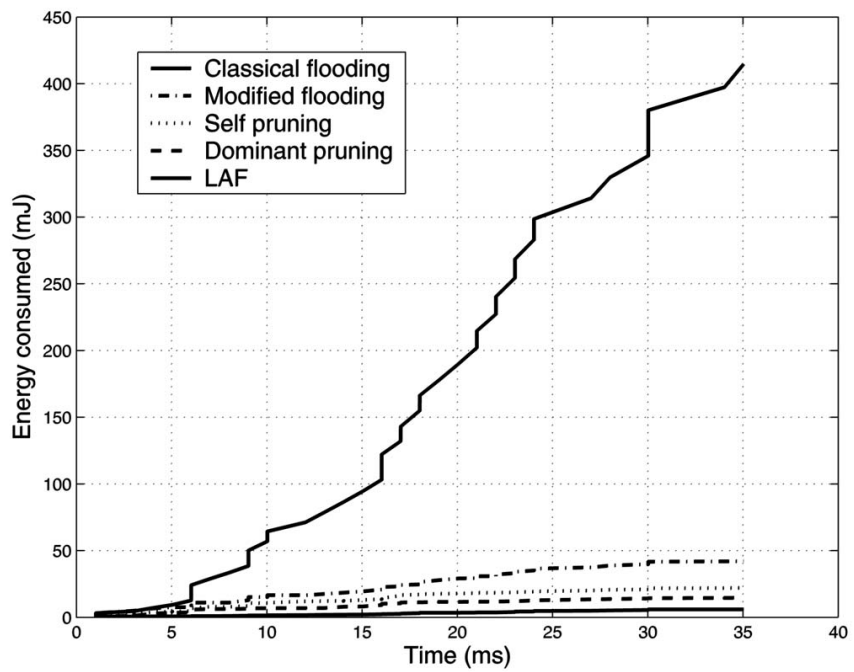

Fig. 9. Energy consumption for different data dissemination methods.

follows: With a small number of virtual grids, energy savings due to the forwarding of the state information in the packet get compensated by the increase in packet length. For a large number of virtual grids, the packet length remains within limits and the energy savings are significant. However, when the number of virtual grids is such that there are only a small number of sensor nodes in each virtual grid, the state information carried by the flooded packet within each virtual grid is very small and, consequently, the energy savings reduce.

\subsubsection{Impact of Packet Size on Energy Savings}

Typical packet sizes in a sensor network are 32 bytes, 64 bytes, 96 bytes, and 128 bytes [18]. An increase in the size of the packet that is flooded results in an increase in energy savings. This is shown in Fig. 11, where three different packet sizes of 64 bytes, 96 bytes, and 128 bytes, respectively, are shown. The energy savings are shown as the percentage savings in energy compared to the classical flooding protocol.

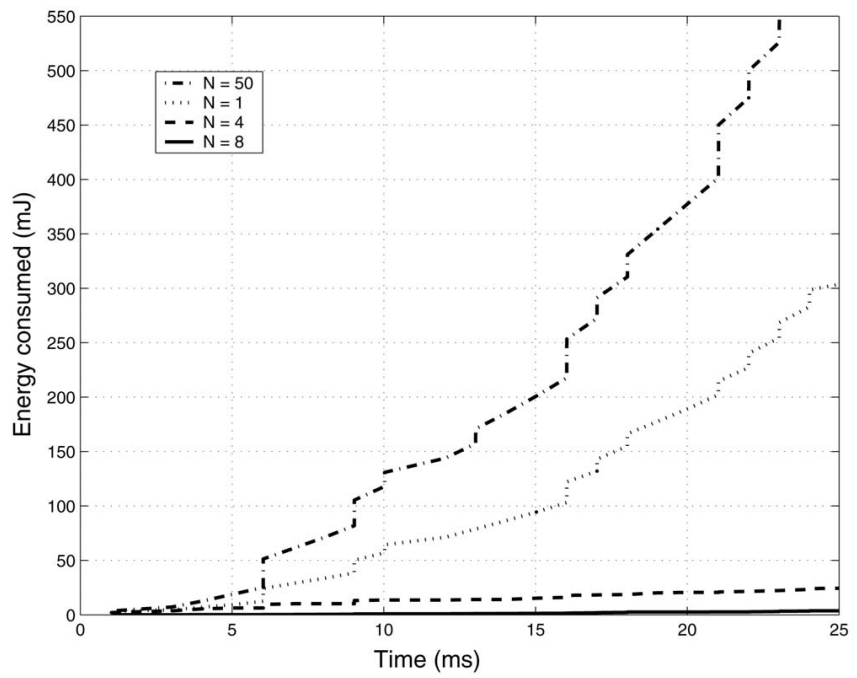

Fig. 10. Effect of number of virtual grids on energy consumption. 


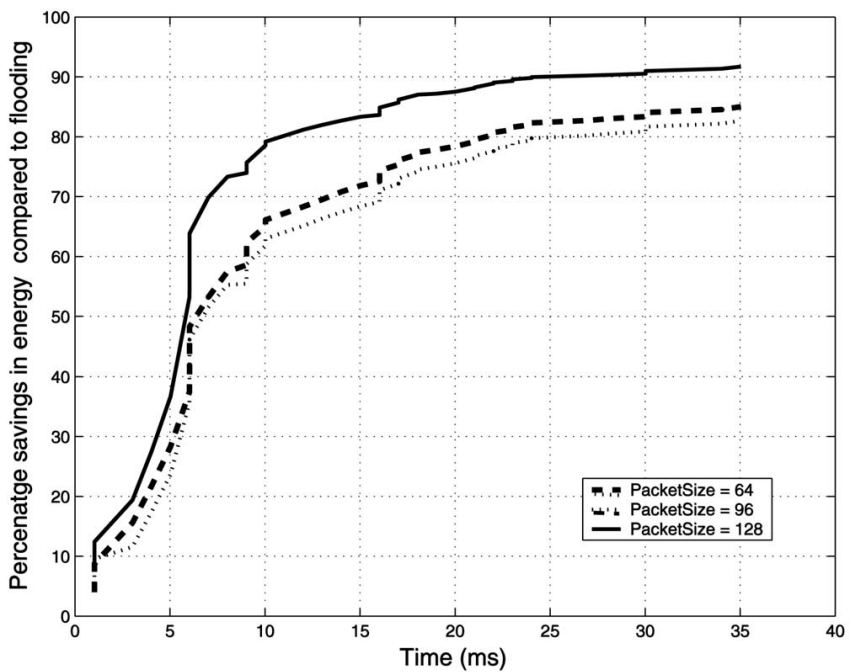

Fig. 11. Energy savings provided by LAF over flooding for different packet sizes.

\subsubsection{Impact of Degree of a Node on Energy Savings}

Fig. 12 shows the effect of the average degree of a node on the energy savings in LAF. A network of 100 nodes is divided into eight grids and the energy consumed for the dissemination of a single 64-byte packet of data to 90 percent, 95 percent, and 99 percent of the nodes is plotted against the average degree of a node. The average degree of a node in the network is varied by changing the locations of the sensors. The total energy dissipated in the network decreases as the average degree of a node increases. This is because a larger number of redundant transmissions are avoided by making use of the information in the Node List.

\subsubsection{Impact of Network Size on LAF}

To study the scalability of network size on LAF, we varied the network size from 100 to 1,000 nodes and flooded a single packet from a randomly selected source. Nodes were randomly deployed on a $200 \times 200 \mathrm{~m}$ grid and the entire

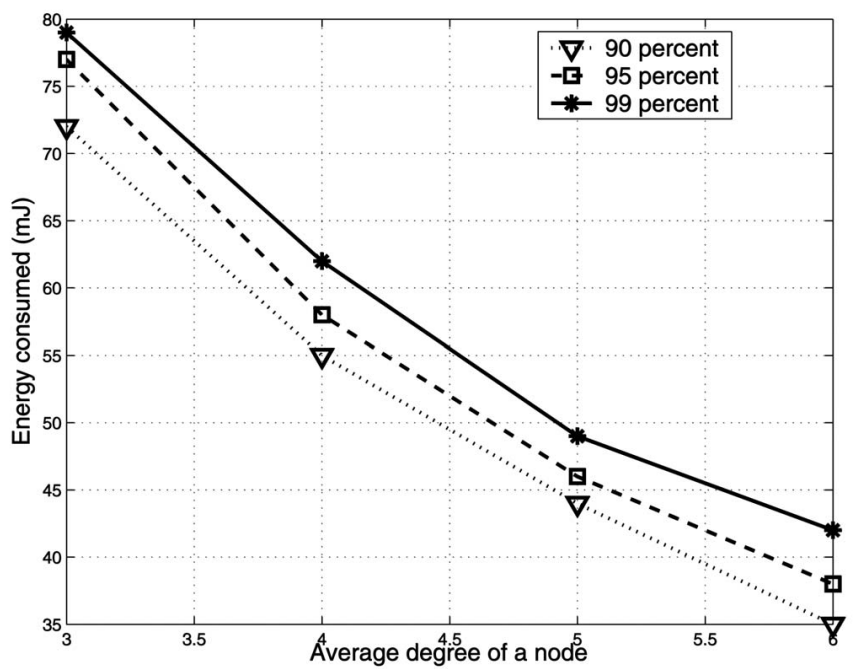

Fig. 12. Effect of average degree of a node on energy consumption.

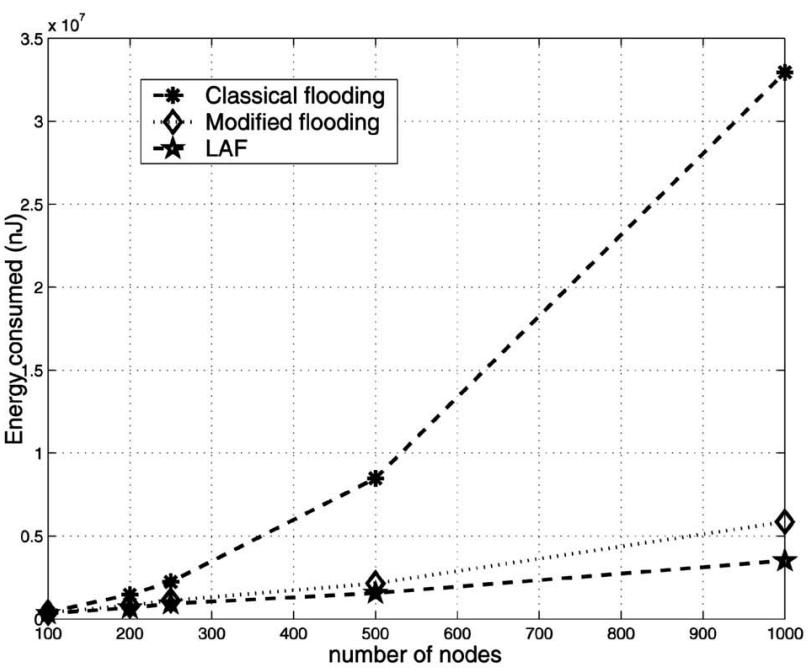

(a)

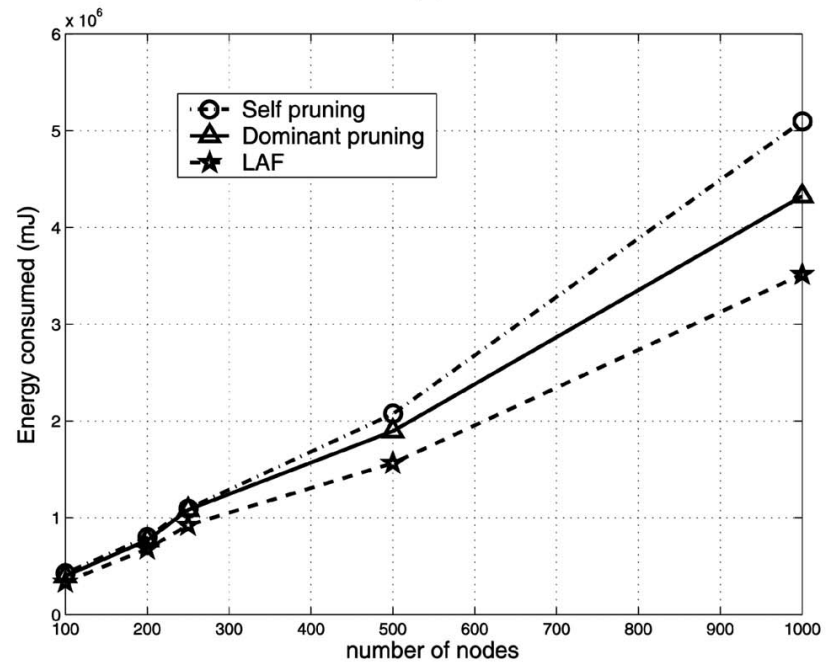

(b)

Fig. 13. Effect of network size on energy consumption for various data dissemination schemes.

grid was divided into eight virtual grids. The results are shown in Fig. 13 and are averaged over 200 simulations. The figure shows that all of the methods are scalable except classical flooding. However, LAF outperforms all the existing methods in terms of energy savings.

\subsubsection{Impact of Error in Location Estimate}

To quantify the effect of error in location estimate on the performance of LAF, we repeated the above simulations by artificially introducing an error in the location estimates of the nodes. We introduced the error by shifting the location of each node by a random amount in the range $[x \pm e, y \pm e]$, where $e$ is the error in the location estimate in terms of the percentage of the radio range of a node and $[x, y]$ is the actual location of a sensor node. Nodes use these artificial locations rather than their actual locations to associate themselves with the virtual grids and determine their type. We found in our simulations that up to 10 percent error in location estimate has negligible impact on the energy efficiency or the latency of LAF for data dissemination. 


\section{Conclusions}

We have presented a new energy-efficient flooding algorithm, termed LAF, for data dissemination in wireless sensor networks. The proposed approach uses the concept of virtual grids to divide the monitored area and nodes then selfassemble into groups of gateway nodes and internal nodes. It exploits the location information available to sensor nodes to prolong the lifetime of sensor network by reducing the redundant transmissions that are inherent in flooding.

We are currently investigating the effect of using nonuniform grid sizes on the energy savings of LAF. Although, in the above discussion, we assumed a lossless network, LAF protocol can be easily adapted to lossy networks. A node can use the knowledge about the quality of a link to its neighbor and rebroadcast the packet multiple times.

Our results raise several interesting questions. First, we have used a uniform square grids in our simulations. However, a nonuniform grid size might be more desirable in situations where the node deployment is inherently nonuniform. Second, the size of the virtual grid can be tailored to the application and be adaptive to the activity in the network. Third, it is important to develop techniques that can dynamically reconfigure the virtual grid in a distributed manner after node failures, wearout, and battery depletion. Finally, as part of future work, the energy savings need to be evaluated on physical hardware to demonstrate the usefulness of LAF.

\section{ACKNOWLEDGMENTS}

This research was sponsored in part by the US Office of Naval Research (ONR) under grant no. N00014-01-1-0712. It was also supported by the US Defense Aadvanced Research Projects Agency and administered by the US Army Research Office under Emergent Surveillance Plexus MURI Award No. DAAD19-01-1-0504. Any opinions, findings, and conclusions or recommendations expressed in this publication are those of the authors and do not necessarily reflect the views of the sponsoring agencies.

\section{REFERENCES}

[1] D. Agrawal, A. El Abbadi, and R. Steinke, "Epidemic Algorithms in Replicated Databases," Proc. ACM Symp. Principles of Database Systems, pp. 161-172, 1997.

[2] I.F. Akyildiz, W. Su, Y. Sankarasubramaniam, and E. Cayiri, "Wireless Sensor Networks: A Survey," Computer Networks, vol. 38, pp. 393-422, Mar. 2002.

[3] J. Albowicz, A. Chen, and L. Zhang, "Recursive Position Estimation in Sensor Networks," Proc. Int'l Conf. Network Protocols, pp. 35-41, 2001.

[4] "Ash Transceiver's Designers Guide," http://www.rfm.com, 2004.

[5] P. Bergamo and G. Mazzini, "Localization in Sensor Networks with Fading and Mobility," Proc. 13th IEEE Int'l Symp. Personal, Indoor, and Mobile Radio Comm., pp. 750-754, 2002.

[6] K.P. Birman, M. Hayden, O. Ozkasap, Z. Xiao, S. Ni, Y. Tseng, Y. Chen, and J. Sheu, "The Broadcast Storm Problem in a Mobile Ad Hoc Network," Proc. ACM/IEEE Int'l Conf. Mobile Computing and Networking, pp. 151-162, Aug. 1999.

[7] K.P. Birman, M. Hayden, O. Ozkasap, Z. Xiao, M. Budiu, and Y. Minsky, "Bimodal Multicast," ACM Trans. Computer Systems, vol. 17, pp. 41-88, May 1999.

[8] J. Broch, D. Maltz, D. Johnson, Y. Su, and J. Jetcheva, "A Performance Comparison of Multi-Hop Wireless Ad Hoc Network Routing Protocols," Proc. ACM/IEEE Int'l Conf. Mobile Computing and Networking, pp. 85-97, 1998.
[9] R.R. Brooks, P. Ramanathan, and A.A. Sayeed, "Distributed Target Classification and Tracking in Sensor Networks," Proc. IEEE, vol. 91, pp. 1163-1171, Aug. 2003.

[10] R.R. Brooks and S.S. Iyengar, Multi-Sensor Fusion: Fundamentals and Applications with Software. Prentice Hall, 1997.

[11] B. Chen, K. Jamieson, H. Balakrishnan, and R. Morris, "Span: An Energy-Efficient Co-Ordination Algorithm for Topology Maintenance in Ad Hoc Wireless Networks," Proc. ACM/IEEE Int'l Conf. Mobile Computing and Networking, pp. 85-96, 2001.

[12] T. Clouqueur, K.K. Saluja, and P. Ramanathan, "Fault Tolerance in Collaborative Sensor Networks for Target Detection," IEEE Trans. Computers, vol. 53, pp. 320-333, Mar. 2004.

[13] D. Estrin, R. Govindan, J. Heidemann, and S. Kumar, "Next Century Challenges: Mobile Networking for Smart Dust," Proc. ACM Conf. Mobile Computing and Networking, pp. 271-278, 1999.

[14] L. Girod and D. Estrin, "Robust Range Estimation for Localization in Ad Hoc Sensor Networks," UCLA CS-TR-2000XX, 2000.

[15] Z. Haas, J. Halpern, and L. Li, "Gossip Based Ad Hoc Routing," Proc. IEEE Infocom Conf., pp. 1702-1706, 2002.

[16] B. Hoffman-Wellenhof, H. Lichteneger, and J. Collins, Global Positioning System: Theory and Practice, fourth ed. Vienna: SpringerVerlag, 1997.

[17] C. Huitema, Routing in Internet. Prentice Hall, 1996.

[18] C. Intanagonwiwat, R. Govindan, and D. Estrin, "Directed Diffusion: A Scalable And Robust Communication Paradigm for Wireless Sensor Networks," Proc. ACM/IEEE Int' Conf. Mobile Computing and Networking, pp. 56-67, 2000.

[19] J. Jetcheva, Y. Hu, D. Maltz, and D. Johnson, "A Simple Protocol for Multicast and Broadcast in Wireless Ad Hoc Networks," Internet Draft: draft-ietf-manet-simple-mbcast-01.txt, July 2001.

[20] R. Kannan, S. Sarangi, S.S. Iyengar, and L. Ray, "Sensor-Centric Quality of Routing In Sensor Networks," Proc. IEEE Infocom, pp. 693-701, 2003.

[21] B. Krishnamachari and S.S. Iyengar, "Distributed Bayesian Algorithms for Fault-Tolerant Event Region Detection in Wireless Sensor Networks," IEEE Trans. Computers, vol. 53, pp. 241-250, Mar. 2004.

[22] J. Kulik, W.R. Heinzelman, and H. Balakrishnan, “NegotiationBased Protocols for Disseminating Information in Wireless Sensor Networks," Wireless Networks, vol. 8, pp. 169-185, 2002.

[23] T.J. Kwon, M. Gerla, V.K. Varma, M. Barton, and T.R. Hsing, "Efficient Flooding with Passive Clustering-An Overhead-Free Selective Forward Mechanism for Ad Hoc/Sensor Networks," Proc. IEEE, vol. 91, pp. 1210-1220, Aug. 2003.

[24] H. Lim and C. Kim, "Multicast Tree Construction and Flooding in Wireless Ad Hoc Networks," Proc. ACM Modeling, Analysis, and Simulation of Wireless and Mobile Systems, pp. 61-68, 2000.

[25] J. Moy, OSPF Version 2, RFC 1583, http://www.ietf.org/rfc/ rfc1583.txt, 1991.

[26] D.C. Oppen and Y.K. Dalal, "The Clearinghouse: A Decentralized Agent for Locating Named Objects in a Distributed Environment," ACM Trans. Office Information Systems, vol. 1, pp. 230-253, July 1983.

[27] N. Patwari and R.J. O'Dea, "Relative Location in Wireless Networks," Proc. IEEE Vehicular Technology Conf., pp. 1149-1153, 1991.

[28] A. Pelt, "Fault-Tolerant Broadcasting and Gossiping in Communication," Networks, vol. 3, pp. 143-156, Oct. 1996.

[29] W. Peng and X. Lu, "On the Reduction of Broadcast Redundancy in Mobile Ad Hoc Networks," Proc. ACM Int'l Symp. Mobile and Ad Hoc Networking and Computing, pp. 129-130, 2000.

[30] V. Phipatanasuphorn and P. Ramanathan, "Vulnerability of Sensor Networks to Unauthorized Traversal and Monitoring," IEEE Trans. Computers, vol. 53, no. 3, pp. 364-369, Mar. 2004.

[31] G.J. Pottie and W. Kaiser, "Wireless Sensor Networks," Comm. ACM, vol. 43, pp. 51-58, 2000.

[32] A. Qayyum, L. Viennot, and A. Laouiti, "Multipoint Relaying for Flooding Broadcast Messages in Mobile Wireless Networks," Proc. IEEE Hawaii Int'l Conf. System Sciences (HICSS), pp. 3898-3907, 2002.

[33] H. Qi, Y. Xu, and X. Wang, "Mobile-Agent-Based Collaborative Signal and Information Processing in Sensor Networks," Proc. IEEE, vol. 91, pp. 1172-1183, Aug. 2003.

[34] C. Raghavendra and S. Singh, "PAMAS: Power-Aware MultiAccess Protocol with Signaling for Ad Hoc Networks," ACM Comm. Rev., pp. 5-26, July 1998. 
[35] A. Savvides, C.-C. Han, and M. Srivastava, "Dynamic FineGrained Localization in Ad Hoc Networks of Sensors," Proc. Int'l Con. Mobile Computing and Networking, pp. 166-179, 2001.

[36] C. Schurgers, V. Tsiatsis, and M. Srivastava, "STEM: Topology Management for Energy-Efficient Sensor Networks," Proc. IEEE Aerospace Conf., pp. 135-145, 2002

[37] K. Sohrabi and G.J. Pottie, "Performance of a Novel Self Organization Protocol for Wireless Ad Hoc Sensor Networks," Proc. IEEE Vehicular Technology Conf., pp. 1222-1226, 1999.

[38] K. Whitehouse and D. Culler, "Calibration as Parameter Estimation in Sensor Networks," Proc. ACM Int'l Workshop Sensor Networks and Applications, pp. 59-67, 2002.

[39] R. Williams, The Geometrical Foundation of Natural Structure: A Source Book of Design. New York: Dover Publications, 1979.

[40] J. Wu and F. Dai, "Broadcasting in Ad Hoc Networks Based on Self-Pruning," Proc. Infocom, pp. 2240-2250, 2003.

[41] Y. Xu, J. Heidemann, and D. Estrin, "Geography Informed Energy Conservation for Ad Hoc Routing," Proc. ACM/IEEE Int'l Conf. Mobile Computing and Networking, pp. 70-84, July 2001.

[42] Y. Xu, J. Heidemann, and D. Estrin, "Adaptive Energy Conservating Routing for Multihop Ad Hoc Routing," Technical Report 527, USC/ISI, Oct. 2000

[43] Wireless LAN Medium Access Control and Physical Layer Specifications, IEEE 802.11 Standard (IEEE CS LAN MAN Standards Committee), Aug. 1999.

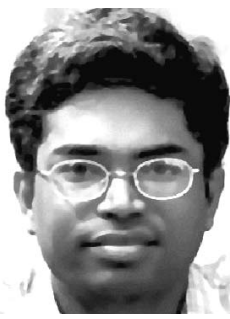

Harshavardhan Sabbineni received the BTech degree in electrical engineering from the Indian Institute of Technology, Kharagpur, India, in 2000 , and the MS degree in electrical and computer engineering from Duke University, Durham, North Carolina, in 2004. He is a PhD candidate in electrical and computer engineering at Duke University. His research interests focus on ad hoc networking, wireless sensor networks, and mobile computing. He is a member of the IEEE.

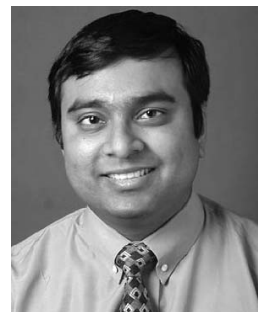

Krishnendu Chakrabarty received the BTech degree from the Indian Institute of Technology, Kharagpur, in 1990, and the MSE and PhD degrees from the University of Michigan, Ann Arbor, in 1992 and 1995, respectively, all in computer science and engineering. He is now an associate professor of electrical and computer engineering at Duke University. During 20002002, he was also a Mercator Visiting Professor at the University of Potsdam in Germany. He is a recipient of the US National Science Foundation Early Faculty (CAREER) award, the US Office of Naval Research Young Investigator award, and the Humboldt Research Fellowship from the Alexander von Humboldt Foundation, Germany. His current research projects include: design and testing of system-on-chip integrated circuits, embedded realtime systems, distributed sensor networks, modeling, simulation and optimization of microfluidic systems, and microfluidics-based chip cooling. $\mathrm{He}$ is a coauthor of two books: Microelectrofluidic Systems: Modeling and Simulation (CRC Press, 2002) and Test Resource Partitioning for System-on-a-Chip (Kluwer, 2002), and the editor of SOC (System-on-a-Chip) Testing for Plug and Play Test Automation (Kluwer 2002). He has contributed chapters to a number of edited books and published more than 160 papers in journals and refereed conference proceedings. He holds a US patent in built-in self-test. $\mathrm{He}$ is a recipient of a best paper award at the 2001 Design, Automation, and Test in Europe (DATE) Conference. He is an associate editor of the IEEE Transactions on Computer-Aided Design of Integrated Circuits and Systems, an editor of the Journal of Electronic Testing: Theory and Applications (JETTA), and a member of the editorial board for Sensor Letters and the Journal of Embedded Computing. He has also served as an associate editor of the IEEE Transactions on Circuits and Systems II: Analog and Digital Signal Processing. $\mathrm{He}$ is a senior member of the IEEE, a member of the ACM and ACM SIGDA, and a member of Sigma $X i$. He serves as vice chair of technical activities on the IEEE's Test Technology Technical Council and is a member of the program committees of several IEEE/ACM conferences and workshops.

$\triangleright$ For more information on this or any other computing topic, please visit our Digital Library at www.computer.org/publications/dlib. 